\title{
Neonatal mortality in South Africa: How are we doing and can
}

\author{
we do better?
}

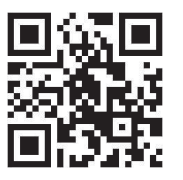

Worldwide there has been a call for a twothirds reduction in childhood mortality by 2015 . Unfortunately, Millennium Development Goal 4 (MDG4) is unlikely to be reached in 2 years' time. ${ }^{[1]}$ Of the 130 million babies born every year, 4 million will die in the first 28 days of life, with the highest risk on the first day of life. ${ }^{[2]}$ The highest rates are in sub-Saharan Africa, where little progress has been made in the last 15 years towards reducing the number of deaths. ${ }^{[2]}$

Early neonatal deaths account for $75 \%$ of all neonatal deaths worldwide, due mainly to prematurity (40\%) and complications of asphyxia (23\%). ${ }^{[2,3]}$ Annually 18 million low-birth-weight babies are born ( $14 \%$ of all births), but they account for $60-80 \%$ of neonatal deaths. ${ }^{[2]}$ Late neonatal deaths are principally due to infections (19\%) with congenital abnormalities responsible for $10 \%$ of deaths. ${ }^{[2,3]}$

Although Africa has made good progress towards reducing under5 mortality, many children continue to die from preventable causes. Neonatal deaths account for approximately $40 \%$ of all deaths in children $>5$ years of age in South Africa (SA) ${ }^{[4,5]}$ In 2009, SA was one of 8 countries in which the neonatal mortality rate (NMR) was higher than the baseline in $1990 .^{[3,5]}$ Starting from a rate of 21/1 000 live births in 1998, neonatal mortality needs to be reduced to 7/1 000 live births in 2015 for MDG4 to be attained ${ }^{[5]}$ While Statistics SA (StatsSA), records the NMR for SA as 14/1 000 live births in 2009, the World Health Organization reported it as 19/1 000 live births. ${ }^{[5]}$ From 2001 to 2008, there was no reduction in the NMR as reported by StatsSA. ${ }^{[5]}$

The Saving Babies 2010 - 2011 report reflects the early neonatal death rate as 21/1 000 live births, with the majority of these deaths occurring in the $1000-1499$ g weight category ${ }^{[6]}$ Deaths due to intrapartum asphyxia were reported as being linked to healthcare provider-associated avoidable factors in $44 \%$ of cases. The top 5 health-worker-related factors were: (i) fetal distress monitored but not detected; (ii) fetal distress not monitored and not detected; (iii) no intervention for prolonged second stage of labour; (iv) delays in referring the patient and $(v)$ delay in calling for expert assistance. Deaths due to immaturity had patient-associated avoidable factors in $30 \%$ of cases. The top 5 factors identified being: (i) delay in seeking medical attention during labour; (ii) non-initiation of antenatal care; (iii) booking late in pregnancy; (iv) infrequent visits to antenatal clinics; and $(v)$ inappropriate response to rupture of membranes. Administrative problems contributed to deaths due to immaturity in $22 \%$ of cases, with inadequate facilities, no accessible intensive care unit (ICU) bed with ventilator, lack of transport and inadequate resuscitation equipment reported frequently.

The belief that only high-level technology can improve neonatal outcome is not appropriate in a developing country. Costeffective interventions such as resuscitation of the newborn baby, breastfeeding, kangaroo mother care (KMC) and prevention of hypothermia can dramatically reduce the number of deaths in resource-limited settings. ${ }^{[7]}$ Neonatal resuscitation training has been reported to reduce deaths due to intrapartum asphyxia by $30 \% .{ }^{\left[{ }^{[8]}\right.}$ Improved obstetric care would also contribute to a reduced number of neonatal deaths. The majority of deaths occurred at Community Health Centres (CHCs) or district hospitals, with the poorest quality of care being rendered in district hospitals. ${ }^{[6]}$

A retrospective descriptive audit conducted over a 1-year period (1 January-31 December 2011) at Steve Biko Academic Hospital (SBAH), a tertiary referral hospital in Gauteng, offers important insights into causes of neonatal mortality. The neonatal records of all patients admitted to the Neonatal Intensive Care Unit (NICU) were surveyed. Cause of death and avoidable factors were collected using the perinatal death datasheet of the Perinatal Problem Identification Program (PPIP).

A total of 890 patients were admitted in the neonatal unit. The neonates' weight distribution varied: 500 - $999 \mathrm{~g}$ in $74(8.3 \%)$ patients, 1000 - $1499 \mathrm{~g}$ in 210 (23.6\%), 1500 - $1999 \mathrm{~g}$ in 224 (25.2\%), 2000 - $2499 \mathrm{~g}$ in $102(11.4 \%)$ and $>2500 \mathrm{~g}$ in $280(31.5 \%)$. A quarter of the total admissions were admitted from another facility or from home (outborn neonates). There were 142 deaths (15.6\% of total admissions), of which 97 (68.3\%) occurred in the first 7 days of life (early neonatal deaths) and $45(31.7 \%)$ between 8 and 28 days of life (late neonatal deaths). Outborn neonates contributed $37.9 \%$ to the total deaths.

The total live births occurring in the region were 15675 , of which 890 (5.8\%) were admitted in NICU. The total number of deaths in the Eastern and Central Tshwane region was 213, of which two-thirds occurred at SBAH $(142 / 213 ; 66.6 \%)$. The NMR for the region was 13.6/1 000, compared with 52.1/1 000 for SBAH.

The top 5 primary obstetric causes of death at SBAH included spontaneous preterm labour (38.7\%), fetal anomaly (23.2\%), hypertensive disorders (12\%), intrapartum asphyxia (9.9\%) and antepartum haemorrhage (5.6\%). The top 4 causes of neonatal death at SBAH NICU were immaturity-related (43\%), infections (26.8\%), congenital abnormalities (17.6\%) and hypoxia (11.3\%). This is similar to that recorded for Eastern and Central Tshwane.

In the Eastern and Central Tshwane region, 174 individual avoidable factors were identified by PPIP. When comparing avoidable factors between admissions and those never admitted, patientassociated factors were distributed reasonably evenly between the 2 groups. Common patient-associated factors included: noninitiation of antenatal care; attempted termination of pregnancy; and delay in seeking medical attention during labour. Administrative problems affecting patients born in the tertiary hospital included: inadequate facilities and equipment; lack of transport; and lack of sufficiently trained personnel. For patients who died outside of the referral hospital, the most common problem was absence of an accessible NICU bed with ventilator. Personnel-associated factors identified were diverse and included hospital-acquired infection, delay in referral, antenatal steroids not given, multiple pregnancy not diagnosed, inadequate resuscitation and monitoring, and inadequate management of second stage of labour.

The NMR for Central and Eastern Tshwane district was 13.6/1 000 live births, similar to the rate of 14/1 000 live births in SA in 2009, as reported by StatsSA. ${ }^{[5]}$ Further reduction to 7/1 000 live births by 2015, which is the target number needed to achieve MDG4, is highly unlikely to occur in the next 2 years.

Darmstadt et al. ${ }^{[7]}$ report that cost-effective and inexpensive interventions such as antibiotics for preterm premature rupture of membranes, antenatal corticosteroids, clean delivery practices, resuscitation of the newborn, breastfeeding, prevention of hypothermia and $\mathrm{KMC}$ can reduce neonatal deaths by $41-72 \%$.

The use of antenatal steroids can reduce the incidence of hyaline membrane disease, intra-ventricular haemorrhages and necrotising enterocolitis, and ultimately significantly reduce neonatal deaths ${ }^{[6]}$ The Vermont Oxford Network (VON) reports that at SBAH only 169/276 
(61.2\%) of babies $<33$ weeks gestation received antenatal steroids (73.1\% of inborn patients and only $25 \%$ of outborn infants).

Prevention and active management of hypothermia, starting in the delivery room, also reduces mortality of premature infants significantly. Although hypothermia was documented as an avoidable cause in only 3 of the SBAH patients, $43.2 \%$ of infants $<1500 \mathrm{~g}$ were hypothermic on admission. The simple expedient of covering preterm infants $<1200 \mathrm{~g}$ or $<28$ weeks gestation in polyethylene or plastic immediately after delivery reduces hypothermia. This should be used in conjunction with warm delivery rooms and functioning incubators and radiant warmers.

Adequate resuscitation with proper training of health personnel and access to the correct equipment will reduce deaths due to prematurity and due to asphyxia. Prompt and early referral for lifesaving treatments, such as surfactant for hyaline membrane disease, and therapeutic hypothermia for asphyxia, is critical. This is especially crucial for therapeutic hypothermia, given the specific window of opportunity of just 6 hours.

The responsible use of oxygen should also be promoted at all levels. Adequate monitoring is essential, especially in the premature infant $<32$ weeks gestation and $<1500 \mathrm{~g}$ birth weight, as these infants are at an increased risk for retinopathy of prematurity (ROP), a leading cause of blindness in children. The second Benefits of Oxygen Saturation Targeting trial (BOOST II) recently reported that lower saturations of $85-89 \%$, which reduce the risk of ROP, had a significantly higher rate of death than saturations of $91-95 \% .{ }^{[9]}$

The (un)availability of beds at referral hospitals needs to be addressed. According to VON, in 2011 SBAH NICU admitted more patients to fewer beds than other similar units in the world, even though the number of deliveries was essentially as high (2 728 v. 2613 live births). The number of staff available to look after these patients at SBAH was limited (1 board-certified specialist v. the ideal of 6). SBAH has no nursery beds, but has 16 KMC beds available at Tshwane District Hospital.

\section{Conclusion}

Neonates continue to die in SA. The question needs to be raised whether the third of deaths occurring outside of the SBAH referral hospital is due to limited availability of NICU beds in the region (which probably reflects many similar regions in our country), or inadequate referral systems and problematic transport systems. Simple preventative measures can be undertaken to decrease the mortality rate outside of tertiary care centres. Resuscitation training of healthcare providers, breastfeeding and KMC programmes, and the use of polyethylene wrappings for neonates $<1200 \mathrm{~g}$ are cost-effective measures that can be undertaken in these facilities to improve outcome. The use of non-invasive ventilation at district hospitals can also be implemented, provided that target saturation protocols are in place, especially for preterm infants.
Increasing the number of neonatal beds available, as well as the number of staff to care for these patients, might have a positive impact on reducing the NMR. Outreach programmes to CHCs and district hospitals to improve neonatal resuscitation skills, care of the premature infant and recognition of need for early referral, should be instituted. Community educational programmes regarding healthy pregnancies resulting in healthy babies should be undertaken to improve help-seeking behaviour, improve clinic attendance, and increase awareness of the benefits of free interventions, such as breastfeeding and KMC.

African countries have progressed well towards reaching MDG4, but the progress is insufficient, especially in SA. SA is currently listed as one of the countries that have made no progress towards reducing maternal mortality. Although reaching MDG4 by 2015 might no longer be possible in SA, the focus should be on the implementation of sustainable measures to improve neonatal mortality, and ultimately reducing under-5 mortality.

Acknowledgements. Thank you to Prof. RC Pattinson and Prof. R Green for their input, as well as the SBAH PPIP team for providing the statistical data.

\section{G Lloyd}

\section{T W de Witt}

Division of Neonatology, Department of Paediatrics and Child Health, University of Pretoria, South Africa

\section{Corresponding author: L G Lloyd (lizel.lloyd@up.ac.za)}

\begin{abstract}
1. Rajaratnam JK, Marcus JR, Flaxman A, et al. Neonatal, postneonatal, childhood, and under-5 mortality for 187 countries, 1970-2010: A systematic analysis of progress towards Millennium Development Goal 4. Lancet 2010;375(9370):1988-2008. [http://dx.doi.org/10.1016/S0140-6736(10)60703-9]

2. Lawn JE, Cousens S, Zupan J and Lancet Neonatal Survival Steering Team. 4 million neonatal deaths:

When? Where? Why? Lancet 2005;365(9462).891-900. [http://dx.do.org/10.1016/30140-6736(05)71048-5] Chopra M, Dawatd E, Pattinson R, Fonn S, Lawn JE. Saving the lives of South Africa's mother babies, and children: Can the health system deliver? Lancet 2009;374(9692):835-846. [http://dx.doi.
org/10.1016/S0140-6736(09)61123-5]

4. Baleta A. South Africa takes steps to reduce perinatal mortality. Lancet 2011;377(9774):1303-1304. [http://dx.doi.org/10.1016/S0140-6736(11)60523-0]

5. Velaphi S, Rhoda N. Reducing neonatal deaths in South Africa - are we there yet, and what can be done? S Afr J Child Health 2012;6:67-71.

6. Pattinson RC. Overview. Neonatal deaths. In: Pattinson RC, ed. Saving Babies 2010-2011: Eighth report on perinatal care in South Africa. Pretoria: Tshepesa Press, 2013:2-23.

7. Darmstadt GL, Bhutta ZA, Cousens S, et al. and Lancet Neonatal Survival Steering Team. Evidence-based, cost-effective interventions: How many newborn babies can we save? Lancet 2005;365(9463):977-988. [http://dx.doi.org/10.1016/S0140-6736(05)71088-6]

8. Lee AC, Cousens $\mathrm{S}$, Wall SN, et al. Neonatal resuscitation and immediate newborn assessment and stimulation for the prevention of neonatal deaths: A systematic review, meta-analysis and Delphi estimation of mortality effect. MBC Public Health 2011:11(Suppl 3):S12. [htp /1d and Delphi estimation of mortality effect. MBC Public Health 2011;11(Suppl 3):S12. [http://dx.do 158-11-S3-S12

9. The BOOST II United Kingdom, Australia, and New Zealand Collaborative Groups. Oxygen saturation and outcomes in preterm infants. N Engl J Med 2013;368:2094-2104. [http://dx.doi.org/10.1056 NEJMoa1302298]
\end{abstract}

S Afr Med J 2013;103(8):518-519. DOI:10.7196/SAMJ.7200 